\title{
Analysis of the kinetics of phase and amplitude gratings recorded in azopolymer films
}

\author{
A. A. Freschi, A. D. S. Cortes, D. A. Donatti, and J. Frejlich* \\ Departamento de Física, Instituto de Geociências e Ciências Exatas, Universidade Estadual Paulista \\ (UNESP), Caixa Postal 178, 13500-970, Rio Claro, SP, Brazil \\ *Laboratório de Óptica, Instituto de Física Gleb Wataghin, Universidade Estadual de Campinas \\ (UNICAMP), Caixa Postal 6165, 13083-970, Campinas, SP, Brazil
}

\begin{abstract}
In this work we use a stabilized holographic technique to study both refractive index and absorption gratings recorded in thin films made of Disperse Red 1 (DR1) embedded in an organic polymer matrix (PMMA) deposited on glass substrate. Gratings are recorded by linearly polarized illumination with the interference pattern of two crossing beams. One of the beams is phase modulated and the interference signals between the transmitted and diffracted waves are detected by a tuned lock-in amplifier. The technique allows measuring separately changes of the refractive index and the absorption coefficient during the course of the photoreaction process. The time evolution of the diffraction efficiencies during recording has shown bi-exponential kinetics for both gratings.
\end{abstract}

\section{INTRODUCTION}

The photoisomerization process in azobenzene materials is subject of great interest because of their potential use in different optical applications including signal processing, optical storage, polarization holography, and various types of photonic devices [1]. Photoinduced dichroism and/or birrefringence were already observed in several systems containing azobenzenes [2-4]. The trans-cis isomerization process is reversible, allowing optical information to be recorded, erased and rewritten in real-time.

Azobenzene derivatives have two geometric isomers, the trans and the cis forms. When these materials are irradiated with linearly polarized light, the azo-dye molecules experience successive cycles of trans-cis isomerization and eventually align perpendicular to the irradiating light polarization [1]. Indeed, the light polarization sensitivity of this photoisomerization reaction originates from the anisotropic shape of the azobenzene molecules. The probability of an azo unit for absorbing a photon is proportional to the cosine square of the angle between the transition dipole moment and the polarization direction of the exciting light. The molecules aligned parallel to the light polarization direction have the highest probability of being excited and isomerized. As a result, when starting from isotropically distributed trans isomers, linearly polarized light burns a hole in this angular distribution (angular hole burning). Also, as the main axis of the azo unit is reoriented due to the isomerization, the main axis of the photoinduced cis state will be tilted with respect to the light polarization direction (angular redistribution). Both process lead to an anisotropic distribution of the azo molecules. Therefore, anisotropies are created in the trans and cis concentrations. As the azobenzene trans isomer is generally more stable than the cis isomer, molecules in the cis form may relax back to the trans form by spontaneous thermal back reaction (or by a reverse photoisomerization cycle).

Here we provide experimental results showing that the anisotropies in the trans and cis concentrations have different time constants, which are reflected in the bi-exponential kinetics of the recorded gratings. 


\section{EXPERIMENTAL METHOD}

The general scheme of the holographic setup is illustrated in Fig. 1. Experiments are carried out at the room temperature $T=300 \mathrm{~K}$. Gratings recorded in a thin film made of Disperse Red 1 (DR1) embedded in an organic polymer matrix (PMMA) deposited on glass substrate. The light source is a solid state laser operated at the wavelength $\lambda=532 \mathrm{~nm}$. The coherent and collimated incident waves have equal intensities $\left(I_{0}\right)$, are linearly polarized (TE), and intersect symmetrically in the sample with an incident angle $\theta \approx 15^{\circ}$. The light intensity distribution in the sample has the form of a sinusoidal fringe pattern, which modulates both, the refractive index and the absorption coefficient. The related phase and amplitude gratings have the same origin (the photoisomerization reaction) and are recorded as a consequence of two different anisotropies (in the trans and cis concentrations). Because the photoisomerization reaction is a local process, we can assume that both gratings are in phase (or counterphase) with respect to the interference pattern. Therefore, the expressions for the output voltages $V_{R}$ and $V_{S}$ (that are proportional to the output light intensities) are [5],

$$
\begin{aligned}
& V_{R}=V_{0}\left(1+2 \sqrt{\eta_{A}} \cos \varphi-2 \sqrt{\eta_{P}} \sin \varphi\right) \\
& V_{S}=V_{0}\left(1+2 \sqrt{\eta_{A}} \cos \varphi+2 \sqrt{\eta_{P}} \sin \varphi\right)
\end{aligned}
$$

where $V_{0}=\kappa I_{0}$ is a constant, with $\kappa$ the irradiance-to-voltage conversion factor of the photodetectors, $\eta_{A}$ and $\eta_{P}$ are the diffraction efficiencies of the amplitude and phase gratings, respectively (we assume $\eta_{A}<<1$ and $\left.\eta_{P}<<1\right)$ and $\varphi$ represents a phase shift perturbation. The bulk absorption effect as well as reflection losses are not considered. One of the input waves is phase modulated with a small amplitude $(\psi=0.4 \mathrm{rad})$ and fast $(\omega=2 \pi \times 1600 \mathrm{rad} / \mathrm{s})$ sinusoidal signal. Due to this fast modulation, phase $\varphi=\psi \sin (\omega t)$, and the Fourier spectrum of the voltage $V_{S}$ (and $V_{R}$ ) presents several harmonic terms of the fundamental frequency $\omega$, where the first and the second ones are,

$$
\begin{gathered}
V_{S}^{(\omega)}=4 V_{0} J_{1}(\psi) \sqrt{\eta_{P}} \sin \omega t \\
V_{S}^{(2 \omega)}=4 V_{0} J_{2}(\psi) \sqrt{\eta_{A}} \cos 2 \omega t .
\end{gathered}
$$

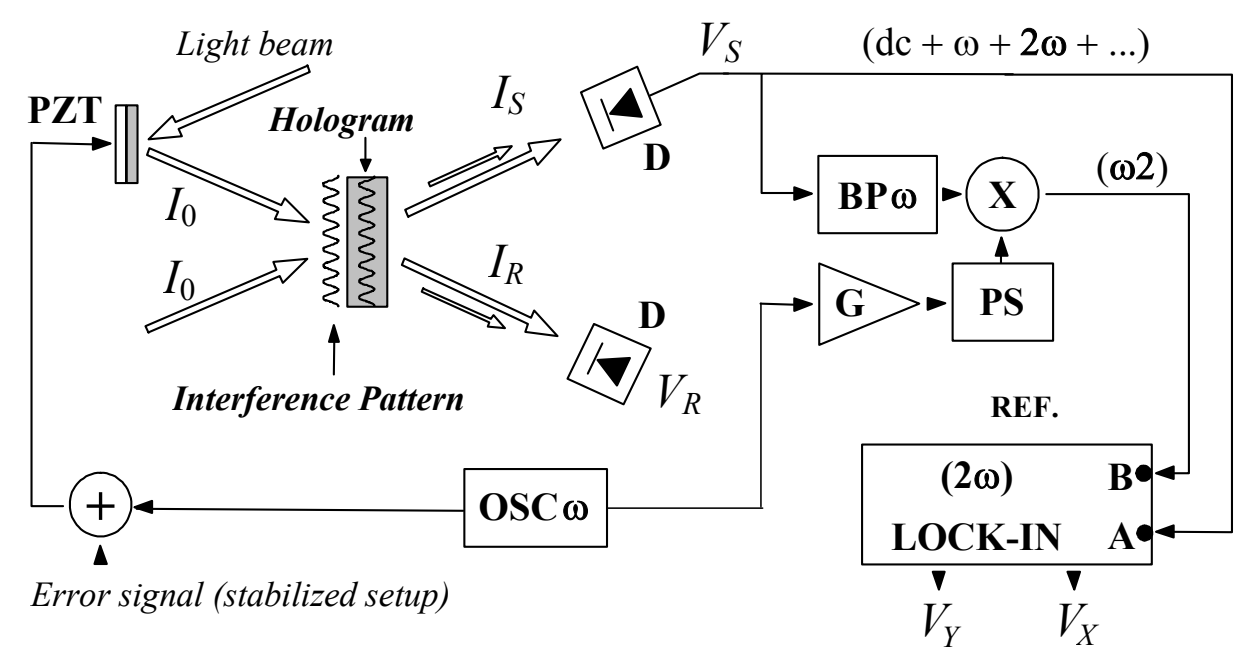

FIGURE 1: Opto-electronic scheme. PZT: piezoelectric supported mirror; D: photodetector; BP: band-pass filter; $\otimes$ : multiplier; G: amplifier; PS: phase-shifter; OSC: sinusoidal oscillator operating at the angular frequency $\omega$; LOCK-IN: two-phase lock-in amplifier tuned to $2 \omega$; A, B: lock-in inputs; $V_{X}, V_{Y}$ : demodulated output signals. 
In Fig. 1, voltage $V_{S}$ is split in two branches: One of them is sent directly to the "A" input of a $2 \omega$-tuned lock-in amplifier; In the other branch, the $\omega$-component, $V_{S}{ }^{(\omega)}$, is filtered out and frequency doubled by mixing it with a sinusoidal wave from the same oscillator that drives the PZT. The mixed signal (called $\omega 2$ component) is sent to the " $\mathrm{B}$ " input of the (two-phase) lock-in amplifier, which is operated in the "A-B" input mode. The amplitude and phase of the $\omega 2$-component can be adjusted [6] so that the $X$ and $Y$ outputs of the lock-in amplifier can be written as,

$$
\begin{aligned}
& V_{X}=2 \sqrt{2} V_{0} J_{2}(\psi) \sqrt{\eta_{P}} \\
& V_{Y}=2 \sqrt{2} V_{0} J_{2}(\psi) \sqrt{\eta_{A}} .
\end{aligned}
$$

Substituting $J_{2}(0.4)=0.0196$ and measuring voltages $V_{0}, V_{X}$, and $V_{Y}$, the diffraction efficiencies can be computed as,

$$
\sqrt{\eta_{A}}=18 V_{Y} / V_{0} \text {, and } \sqrt{\eta_{P}}=18 V_{X} / V_{0}
$$

\section{RESULTS AND CONCLUSIONS}
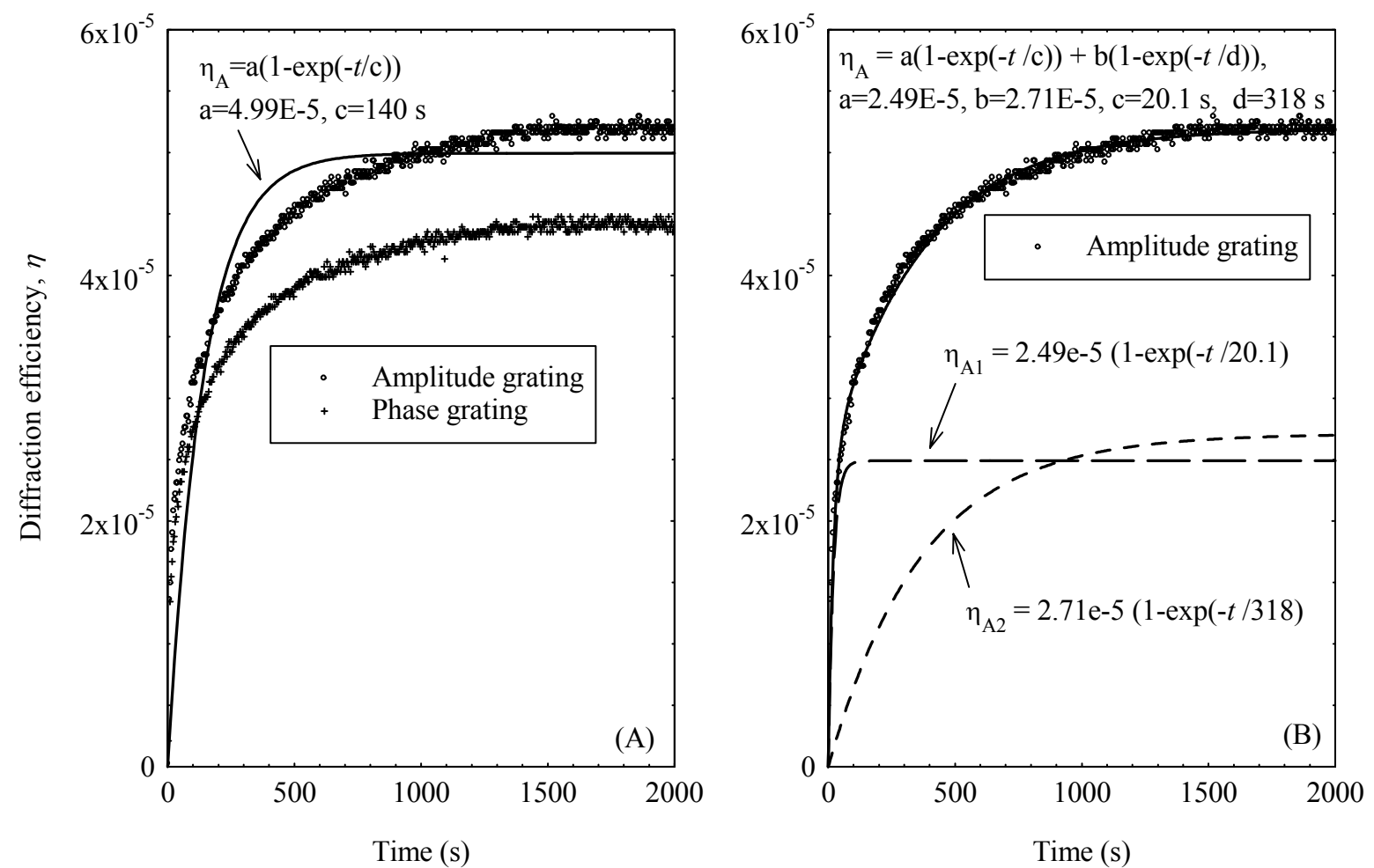

FIGURE 2: Experimental results (dots), showing the time evolution of the amplitude and phase gratings. Fig. 2A) The solid line is mono-exponential curve fitting for the amplitude grating; Fig 2B) The dots represent the amplitude grating and the (superposed) solid line is a bi-exponential curve fitting, with parameters $c$ and $d$ the time constants. The dashed lines show the contributions of the fast $(c)$ and slow $(d)$ components of the grating separately.

Fig. 2 shows the typical time evolution of the amplitude and the phase gratings. We note that when detector $V_{R}$ is used (instead of $V_{S}$ ), the amplitude grating remains unchanged whereas the phase grating 
changes sign, as expected. This confirms that the gratings are not mixed in the results. The sample is assumed to be isotropic initially, with most of the azo molecules in the more stable trans form. The irradiation was started at $t=0$. In the illuminated regions (bright fringes) the trans populations decreases whereas the cis populations increases due to the photoisomerization reaction; these populations remain unaltered in the dark regions. As a result, a spatial modulation of the absorption coefficient (amplitude grating) and of the refractive index (phase grating) takes place. The time evolution of both gratings depends on the anisotropies created in the trans and cis forms. One can see clearly from Fig. 2 that the gratings don't evolve as a monoexponential function, thanks to the different time constants of the angular hole burning and angular redistribution processes, which lead to a bi-exponential evolution of the diffraction efficiencies (Fig. 2B).

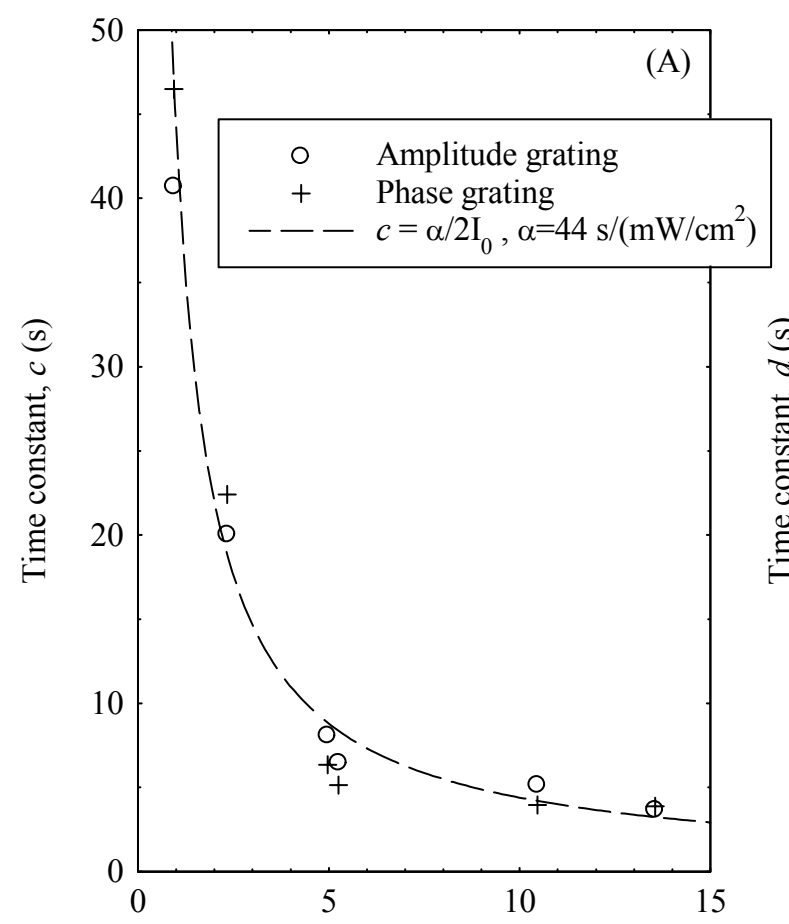

Average light intensity, $2 I_{0}\left(\mathrm{~mW} / \mathrm{cm}^{2}\right)$

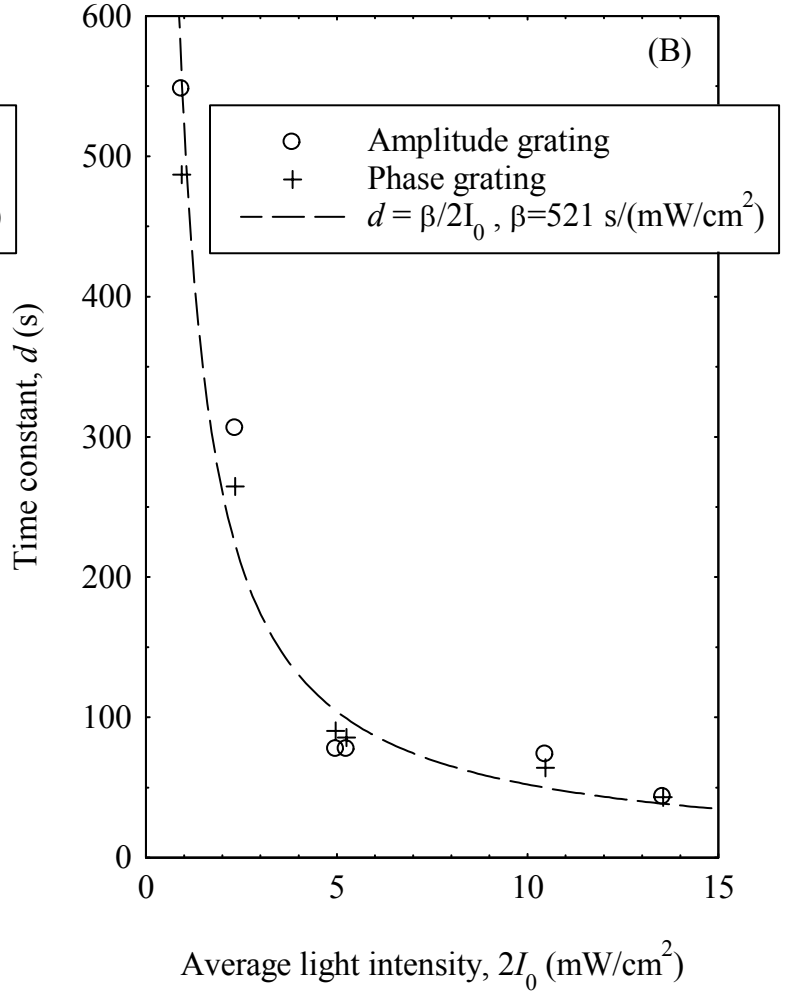

FIGURE 3: Time constants of the fast $(c)$ and slow $(d)$ photoinduced anisotropies versus light intensity. The dashed lines are curve fittings showing that both, $c$ and $d$, are inversely proportional to the average light intensity of the fringe pattern.

Fig. 3 is a plot of the measured time constants for both (amplitude and phase) gratings at different light intensities. It is not surprising the fact that both gratings have similar behaviors because both are originated from the same photoisomerization reaction. It can be seen that the time constants $(c$ and $d)$ are inversely proportional to the light intensity (at least in the investigated region), with $c$ being smaller by approximately one order of magnitude.

In summary, we have investigated the dynamics of phase and amplitude gratings recorded in thin film made of DR1 embedded in an PMMA. It is worth noting that one cannot hope to define characteristic time constants for the photoisomerization reaction processes regardless of the properties of the polymer matrix. Indeed, different kinetics of the photoinduced anisotropies is expected depending on the free volume distribution and the Brownian relaxation [1]. In any case, the results reported here may lead to a physical insight into the photoisomerization reaction and allow getting information on the different steps of the reorientation mechanisms for the trans and cis forms. 
The authors acknowledge financial support of the Fundação de Amparo à Pesquisa do Estado de São Paulo (FAPESP), Brazil, and Conselho Nacional de Desenvolvimento Científico e Tecnológico (CNPq), Brazil.

\section{REFERENCES}

1. Z. Sekkat, J. Wood, and W. Knoll, J. Phys. Chem. 99, 17226-17234 (1995).

2. H. Taunaumang, M. Solyga, M. O. Tija, A. Miniewicz, Thin Solid Films 461, 316-324 (2004).

3. P.-A. Blanche, Ph. C. Lemaire, C. Maertens, P. Dubois, R. Jérome, Opt. Commun. 185, 1-12 (2000).

4. W. Zhang, S. Bian, S. I. Kim, M. G. Kuzyk, Opt. Lett. 27 (13), 1105-1107 (2002).

5. J. Frejlich, A. A. Kamshilin, and P. M. Garcia, Opt. Lett. 17 (4), 249-251 (1992).

6. A. A. Freschi, F. J. dos Santos, E. L. Rigon, and L. Cescato, Opt. Commun. 208, 41-49 (2002). 
Copyright of AIP Conference Proceedings is the property of American Institute of Physics and its content may not be copied or emailed to multiple sites or posted to a listserv without the copyright holder's express written permission. However, users may print, download, or email articles for individual use. 